\title{
Erratum to: Non-Ceruloplasmin Copper Distincts Subtypes in Alzheimer's Disease: a Genetic Study of ATP7B Frequency
}

\author{
Rosanna Squitti ${ }^{1,2}$ - Mariacarla Ventriglia ${ }^{1}$ - Massimo Gennarelli ${ }^{3,4}$. \\ Nicola A. Colabufo ${ }^{5}$ - Imane Ghafir El Idrissi ${ }^{6}$ - Serena Bucossi ${ }^{2}$. \\ Stefania Mariani ${ }^{1}$ - Mauro Rongioletti ${ }^{7}$ - Orazio Zanetti ${ }^{8} \cdot$ Chiara Congiu $^{8}$. \\ Paolo M. Rossini ${ }^{9} \cdot$ Cristian Bonvicini $^{3}$
}

Published online: 28 January 2016

(C) Springer Science+Business Media New York 2016

\section{Erratum to: Mol Neurobiol}

DOI: 10.1007/s12035-015-9664-6

The original paper of this article unfortunately contains error in the figure legend embedded in Fig. 2.

The colored square indicating the High Non-Cp-Cu AD group has to be in dark blue (and not in light blue) and the colored square indicating the Normal Non-Cp-Cu AD group has to be in light blue (and not in dark blue).

The authors do hereby publish the correct image and legend of Fig. 2.

The online version of the original article can be found at http://dx.doi.org/ 10.1007/s12035-015-9664-6.

\section{Rosanna Squitti} rosanna.squitti@afar.it

Fatebenefratelli Foundation, AFaR Division, Fatebenefratelli Hospital, Isola Tiberina, Rome, Italy

2 Institute of Cognition Sciences and Technologies (CNR), Fatebenefratelli Hospital, Isola Tiberina, Rome, Italy

3 Genetic Unit, IRCCS Centro San Giovanni di Dio, Fatebenefratelli, Brescia, Italy

4 Departement of Molecular and Translational Medicine, University of Brescia, Brescia, Italy
5 Dipartimento di Farmacia-Scienze del Farmaco, Università degli Studi di Bari “A. Moro”, via Orabona 4, 70125 Bari, Italy

6 Biofordrug srl, Spin-off, Università degli studi di Bari “A. Moro”, via Orabona 4, 70125 Bari, Italy

7 Unità Biomolecolare - Fatebenefratelli Hospital, Isola Tiberina, Rome, Italy

8 Alzheimer Unit, IRCCS Centro San Giovanni di Dio, Fatebenefratelli, Brescia, Italy

9 Institute of Neurology, Department of Geriatrics, Neuroscience and Orthopedics, Catholic University, Policlinic A. Gemelli, Rome, Italy 
A

AAC protective ATP7B haplotype for Copper AD

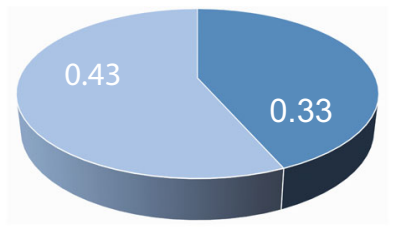

- High Non-Cp-Cu AD group normal Non-Cp-Cu AD group

B

GT risk ATP7B haplotype

for Copper AD

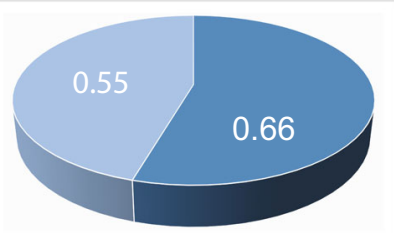

High Non-Cp-Cu AD group

Normal Non-Cp-Cu AD group

Fig. 2 Haplotypes with increased or decreased risk for copper Alzheimer's disease (AD). The pie charts show the frequency distribution (percentage) of the ATP7B haplotypes between the high non- $\mathrm{Cp}-\mathrm{Cu} \mathrm{AD}$ group (dark blue) and the normal non-Cp-Cu AD group (light blue). a Protective haplotype: the $\mathrm{AAC}$ haplotype appears more frequently in AD patients with normal non-Cp-Cu (43 vs. $33 \%$; $\mathrm{Pm}$ values are obtained by 100,000 permutation formultiple test correction, $\mathrm{Pm}=0.03)$. b Risk haplotype for copper AD: the GT haplotype appears more frequently in the higher non- $\mathrm{Cp}-\mathrm{Cu} \mathrm{AD}$ than in normal non- $\mathrm{Cp}-\mathrm{Cu}$ AD group (66 \% vs. normal non-Cp-Cu $55 \%$; OR 1.57; $95 \%$ CI (1.1$2.5) ; \mathrm{Pm}=0.01$ ) (color figure online) 